
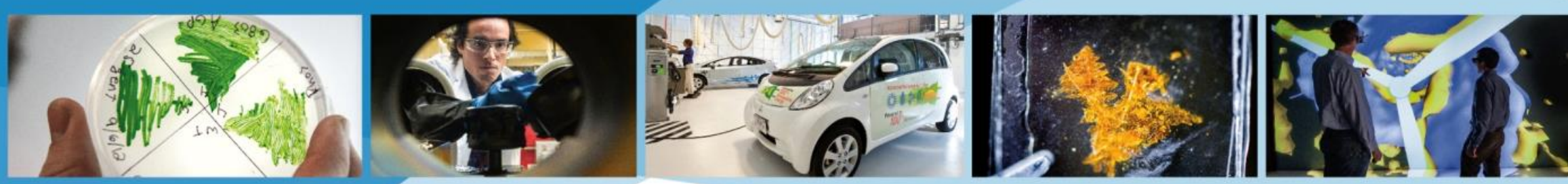

\title{
Heavy-Duty Vehicle Port Drayage Drive Cycle Characterization and Development
} NREL/PR-5400-67291

Robert Prohaska, Arnaud Konan, Kenneth Kelly \& Michael Lammert

\section{SAE 2016 Commercial Vehicle}

Engineering Congress

October 4-6, 2016 


\section{US Department of Energy National Labs}

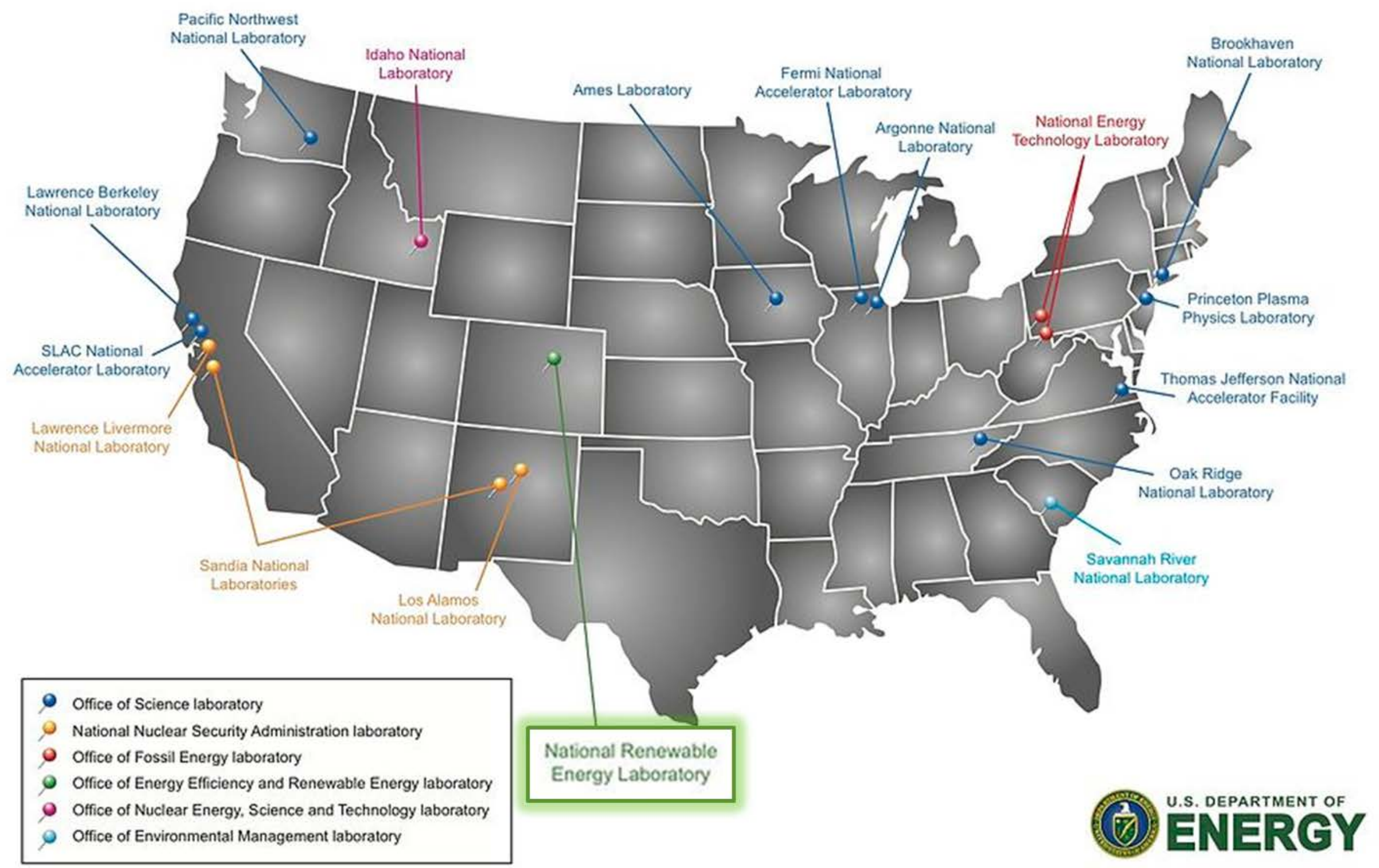




\section{NREL Transportation and Mobility RD\&D Activities}

\section{Advanced Combustion \& Fuels}

- Advanced Petroleum \& Biofuels

- Combustion / Emissions Measurements

- Vehicle, Engine \& Component Testing

\section{Vehicle Fleet Test \& Evaluation}

- Advanced In-Field Data Collection

- Medium / Heavy Duty (MD/HD) Testing \& Analysis

- Drive Cycle Analysis

- Big Data Collection, Storage \& Analysis

- Vehicle Modeling \& Simulation

- Optimization Tools \& Analysis

- MD/HD Dynamometer Testing

\section{Advanced Energy Storage}

- Development, Testing \& Analysis

- Thermal Characterization

- Life Cycle / Abuse Testing \& Modeling

- Electrode Material Development

\section{Regulatory Support}

- EPA Greenhouse Gas Rulemaking

- Data \& Policy Analysis

- Technical Integration

- Fleet Assistance

\section{Vehicle Thermal Management}

- Integrated Thermal Management

- Climate Control/Idle Reduction

- Advanced HVAC

\section{Infrastructure}

- Vehicle-to-Grid Integration

- Renewables

- Charging Equipment \& Controls

- Fueling Stations \& Equipment

- Roadway Electrification 


\section{NREL Medium and Heavy-Duty Fleet Evaluations}

Field Test and Evaluation provides medium-duty (MD) and heavy-duty (HD) test results, aggregated data, and detailed analysis.

- $3^{\text {rd }}$ party unbiased data: Provides data that would not normally be shared by industry in an aggregated and detailed manner

- Over 9 million miles of advanced technology MD and HD truck data have been collected, documented, and analyzed on over 240 different vehicles since 2002

- Data, Analysis, and Reports are shared within DOE, national laboratory partners, and industry for R\&D planning and strategy development

- Results help:

o Guide R\&D for new technology development

o Help define intelligent usage of newly developed technology

o Help fleets/users understand all aspects of advanced technology
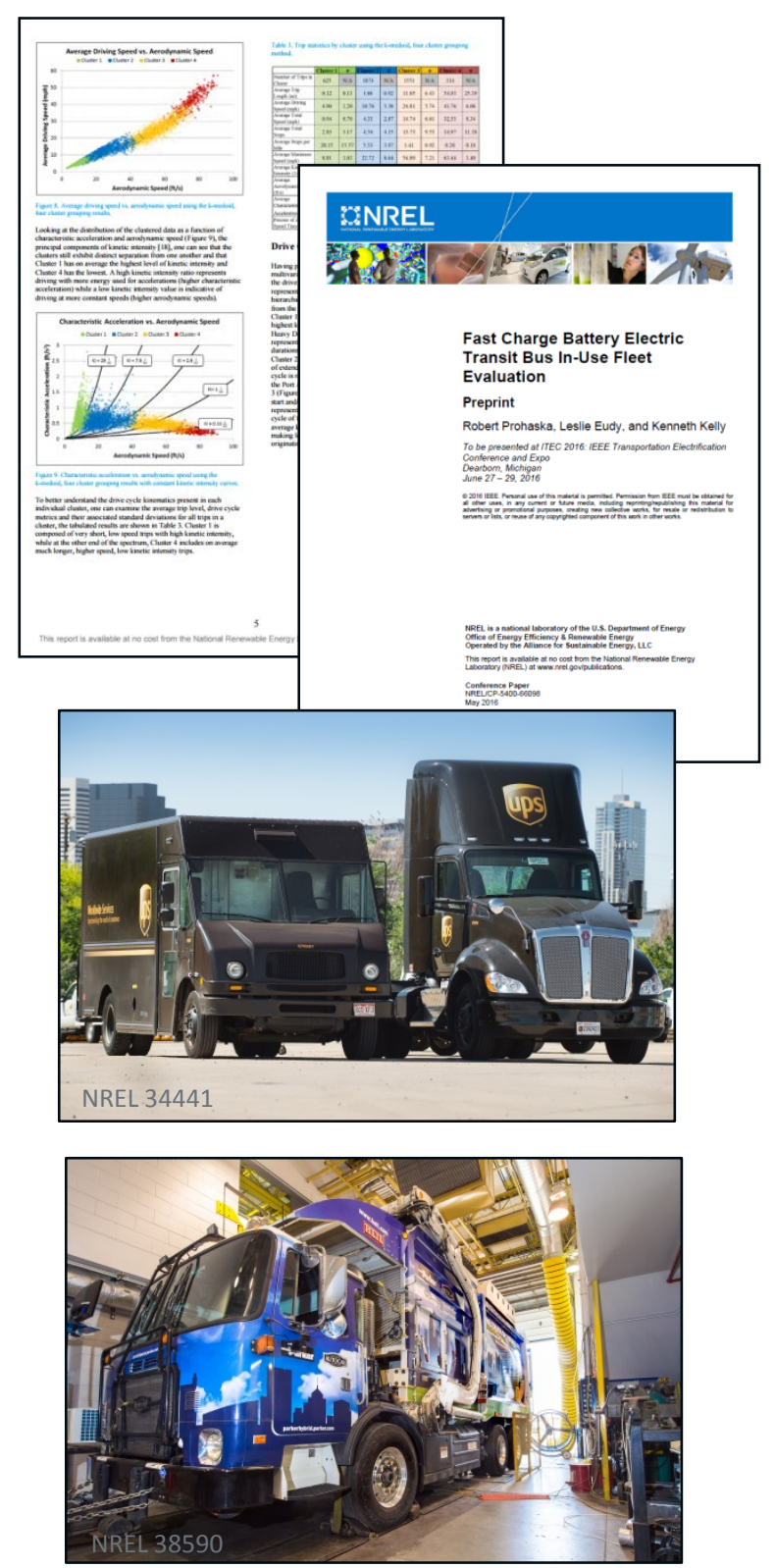


\section{Field Data Collection}

- In support of multiple projects, NREL has collected in-use field data from 30 separate vehicles operating in the ports of Los Angeles and Long Beach (POLA/POLB).

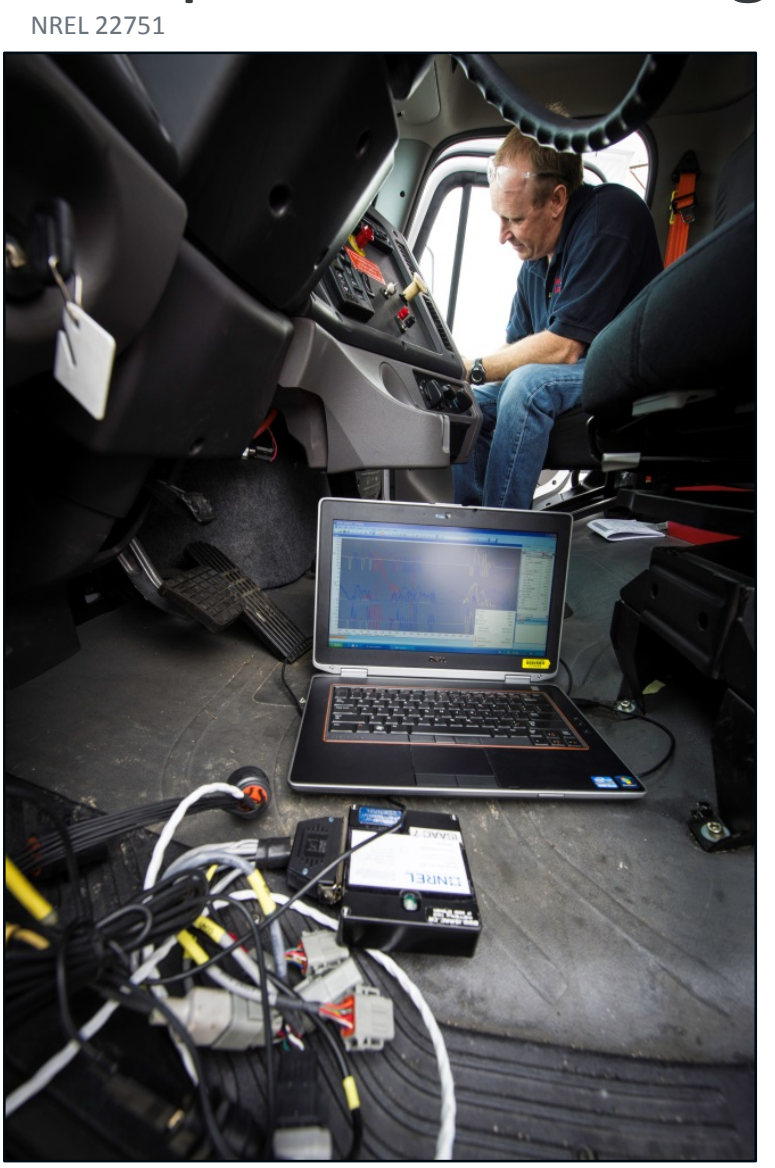

\begin{tabular}{|c|c|}
\hline \multicolumn{2}{|c|}{ Port Drayage Field Data } \\
\hline Total mileage & 36,444 Miles \\
\hline Total hours of operation & 2,809 Hours \\
\hline Driving days & 557 Days \\
\hline Operating companies & 3 Companies \\
\hline Unique vehicles & 30 Vehicles \\
\hline Vehicle manufacturers & $\begin{array}{r}\text { Freightliner, Peterbilt \& } \\
\text { Sterling }\end{array}$ \\
\hline
\end{tabular}




\section{Port of Los Angeles / Port of Long Beach}

- Collectively the two ports form the largest and busiest container port in the United States and the fifth busiest in the world
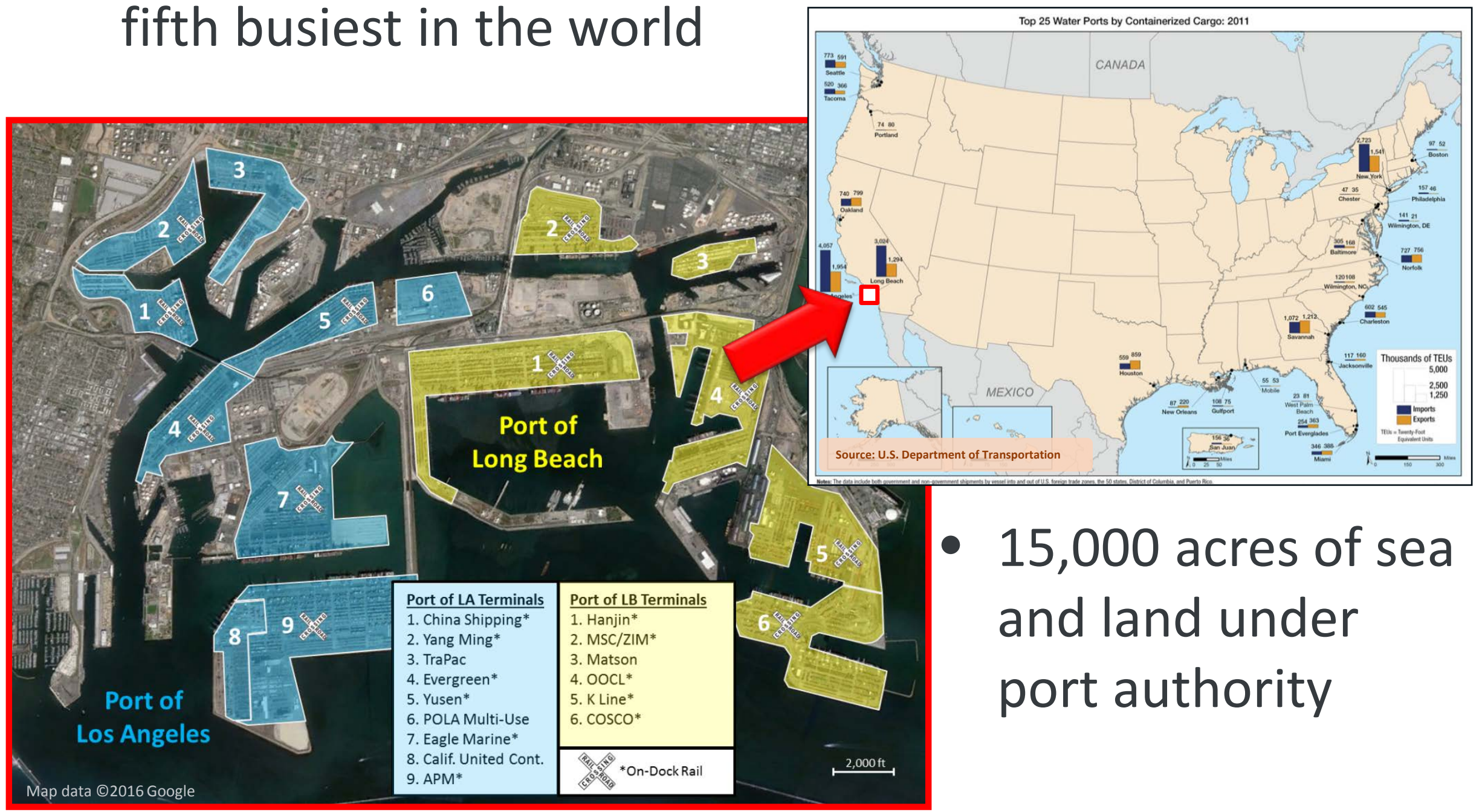


\section{Sample Routes}

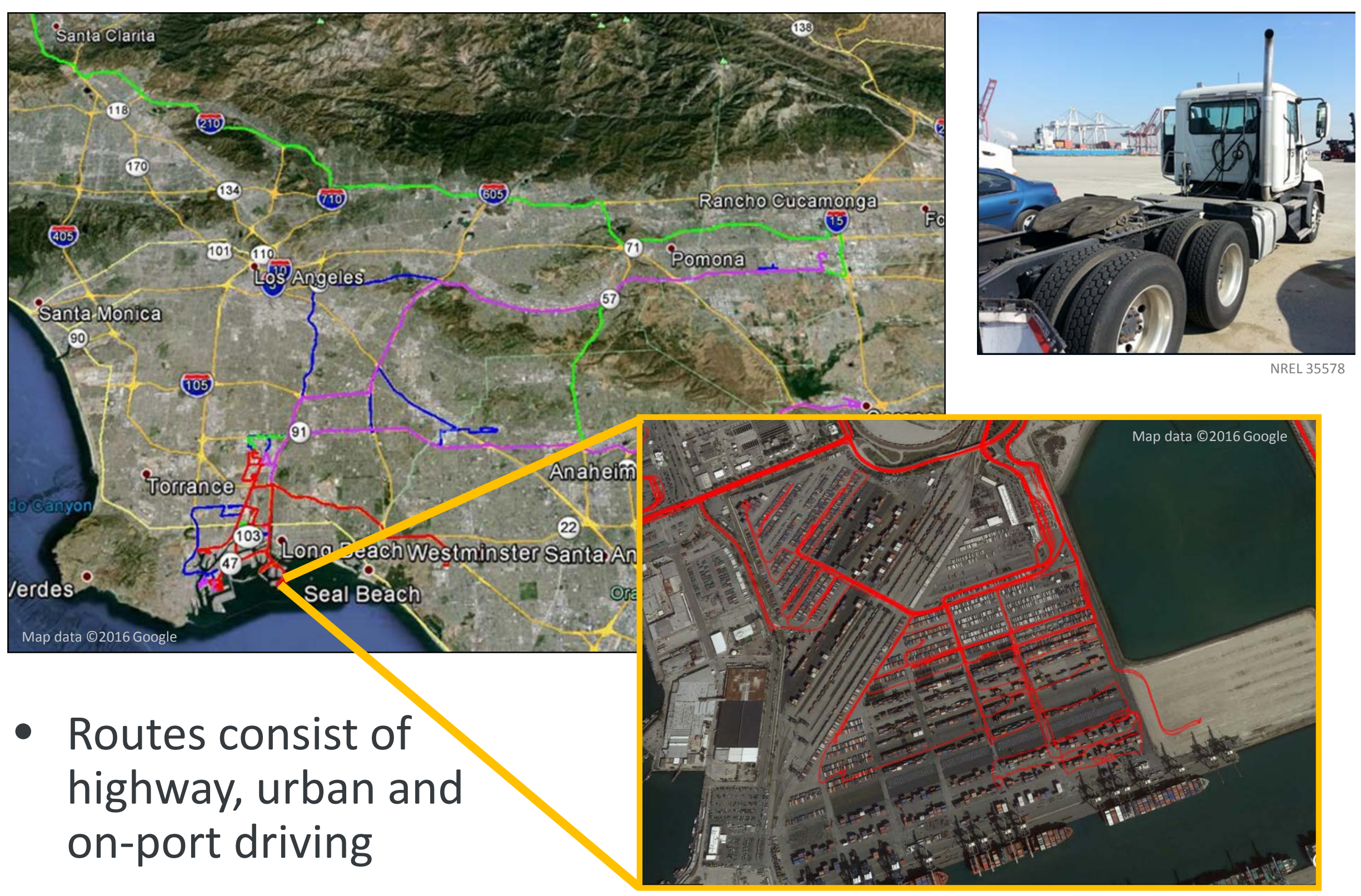




\section{Geospatial Analysis}

- Trip-level geospatial origin and destination analysis demonstrated most activity in one of six combinations:

1) From: Port Area | To: Port Area

2) From: Near Dock | To: Near Dock

3) From: Port Area | To: Near Dock

4) From: Near Dock | To: Port Area

5) From: Near Dock | To: Inland Empire

6) From: Inland Empire | To: Near Dock

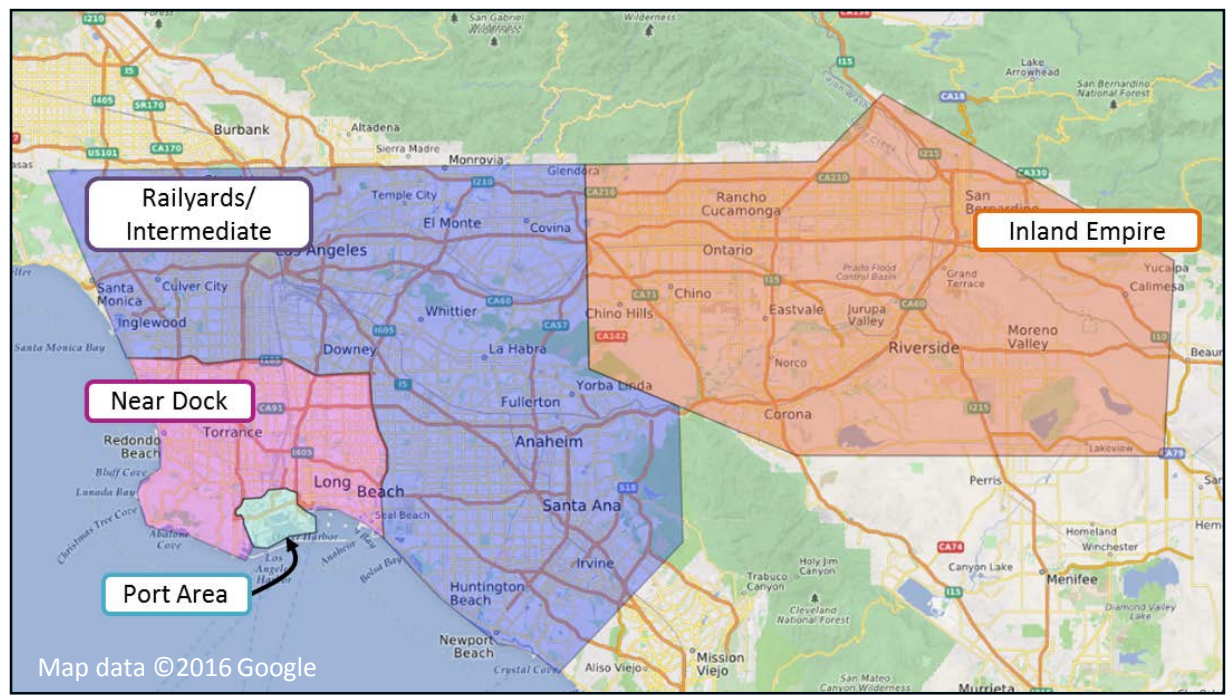

- Trip defined as a key-cycle (on/off) event 


\section{Geospatial Analysis}

- Six of 25 trip combinations include:

o $74 \%$ of the total mileage

o $75 \%$ of the total fuel consumed

\section{o $83 \%$ of the total operating time}
1) From: Port Area | To: Port Area
2) From: Near Dock | To: Near Dock
3) From: Port Area | To: Near Dock
4) From: Near Dock | To: Port Area
5) From: Near Dock | To: Inland Empire
6) From: Inland Empire | To: Near Dock

\begin{tabular}{|l|c|c|c|c|c|}
\hline \multicolumn{7}{|c|}{ Mileage } \\
\hline Fo & Port Area & Near Dock & $\begin{array}{c}\text { Rail } \\
\text { Yard/Inter. }\end{array}$ & $\begin{array}{c}\text { Inland } \\
\text { Empire }\end{array}$ & Other \\
\hline Port Area & $14.9 \%$ & $13.7 \%$ & $1.8 \%$ & $2.1 \%$ & $0.0 \%$ \\
\hline Near Dock & $12.3 \%$ & $10.4 \%$ & $4.6 \%$ & $12.2 \%$ & $1.9 \%$ \\
\hline Rail Yard/Inter. & $3.3 \%$ & $3.1 \%$ & $1.2 \%$ & $0.7 \%$ & $0.0 \%$ \\
\hline Inland Empire & $3.7 \%$ & $10.2 \%$ & $0.7 \%$ & $1.6 \%$ & $0.0 \%$ \\
\hline Other & $0.5 \%$ & $0.9 \%$ & $0.1 \%$ & $0.1 \%$ & $0.1 \%$ \\
\hline
\end{tabular}

\begin{tabular}{|l|c|c|c|c|c|}
\hline \multicolumn{7}{|c|}{ Fuel Consumption } \\
\hline From & Port Area & Near Dock & $\begin{array}{c}\text { Rail } \\
\text { Yard/Inter. }\end{array}$ & $\begin{array}{c}\text { Inland } \\
\text { Empire }\end{array}$ & Other \\
\hline Port Area & $18.1 \%$ & $14.1 \%$ & $2.1 \%$ & $1.9 \%$ & $0.0 \%$ \\
\hline Near Dock & $11.3 \%$ & $10.5 \%$ & $4.8 \%$ & $12.8 \%$ & $2.0 \%$ \\
\hline Rail Yard/Inter. & $2.6 \%$ & $2.8 \%$ & $1.6 \%$ & $0.7 \%$ & $0.0 \%$ \\
\hline Inland Empire & $2.8 \%$ & $\mathbf{8 . 3 \%}$ & $0.6 \%$ & $1.7 \%$ & $0.0 \%$ \\
\hline Other & $0.4 \%$ & $0.7 \%$ & $0.0 \%$ & $0.1 \%$ & $0.2 \%$ \\
\hline
\end{tabular}

\begin{tabular}{|l|c|c|c|c|c|}
\hline \multicolumn{7}{|c|}{ Operating Time } \\
\hline Fo & Port Area & Near Dock & $\begin{array}{c}\text { Rail } \\
\text { Yard/Inter. }\end{array}$ & $\begin{array}{c}\text { Inland } \\
\text { Empire }\end{array}$ & Other \\
\hline Port Area & $\mathbf{2 8 . 2 \%}$ & $\mathbf{1 5 . 0 \%}$ & $0.9 \%$ & $0.7 \%$ & $0.0 \%$ \\
\hline Near Dock & $\mathbf{1 0 . 6 \%}$ & $\mathbf{2 0 . 4 \%}$ & $2.8 \%$ & $\mathbf{4 . 5 \%}$ & $0.7 \%$ \\
\hline Rail Yard/Inter. & $1.6 \%$ & $2.0 \%$ & $3.2 \%$ & $0.3 \%$ & $0.0 \%$ \\
\hline Inland Empire & $1.3 \%$ & $\mathbf{4 . 1 \%}$ & $0.4 \%$ & $2.4 \%$ & $0.0 \%$ \\
\hline Other & $0.3 \%$ & $0.3 \%$ & $0.0 \%$ & $0.1 \%$ & $0.2 \%$ \\
\hline
\end{tabular}




\section{Geospatial Analysis}

- While the vehicles may start and stop their trips in the same region, the trip activity could vary widely

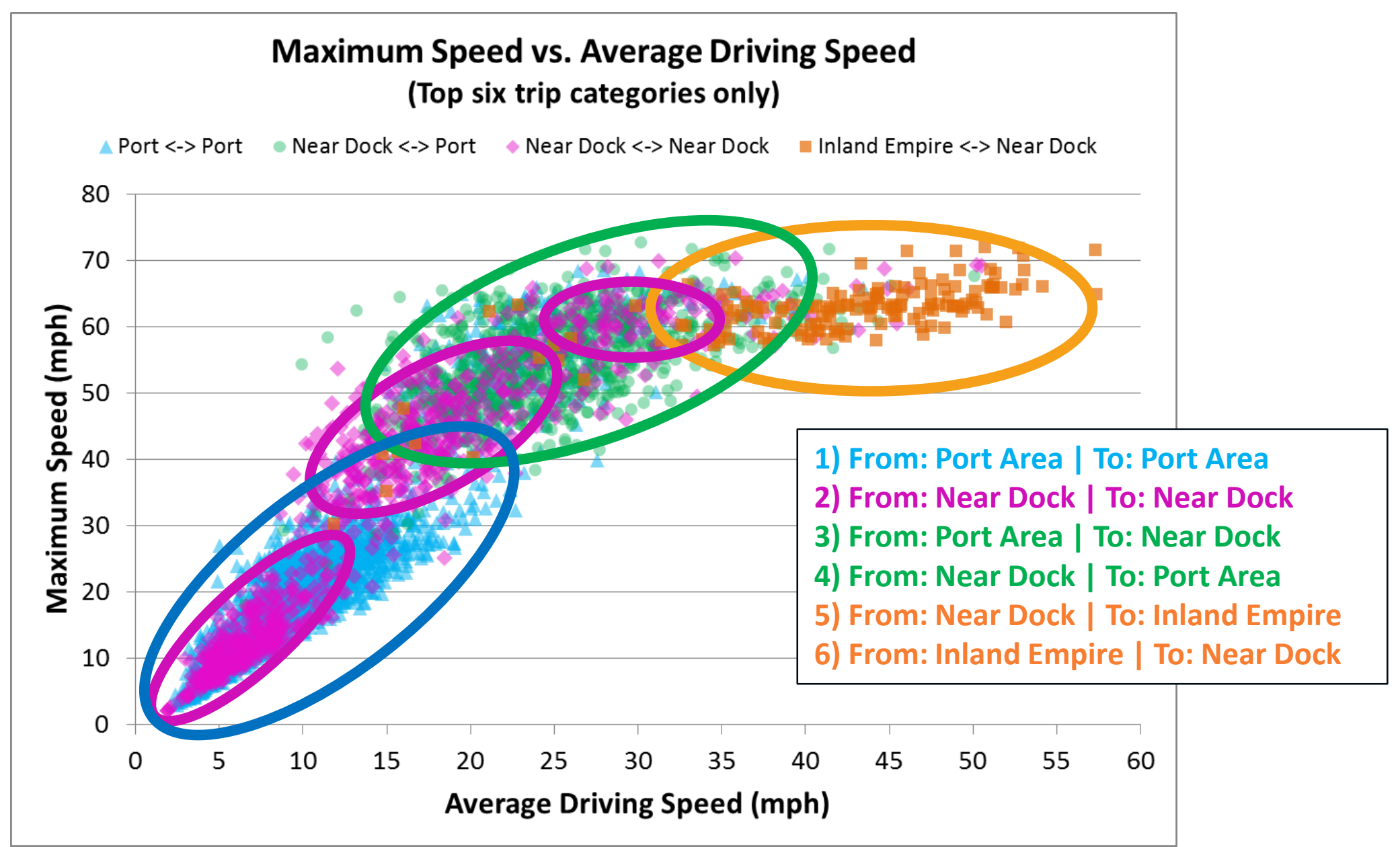




\section{Clustering Analysis}

- Step 1: Trip-level metrics selected to define kinematic driving behavior

- Step 2: Metrics scaled using the z-score scaling method

- Step 3: Identify number of clusters using both mean shift and elbow

- Aerodynamic speed

- Average driving speed

- Characteristic acceleration

- Kinetic intensity

- Maximum speed

- Stops/mile

- Total average speed

- Total distance

- Total stops method

- Step 4: Use k-medoid clustering algorithm

$$
F(x)=\operatorname{minimize} \sum_{i=1}^{n} \sum_{j=1}^{n} d(i, j) z_{i j}
$$

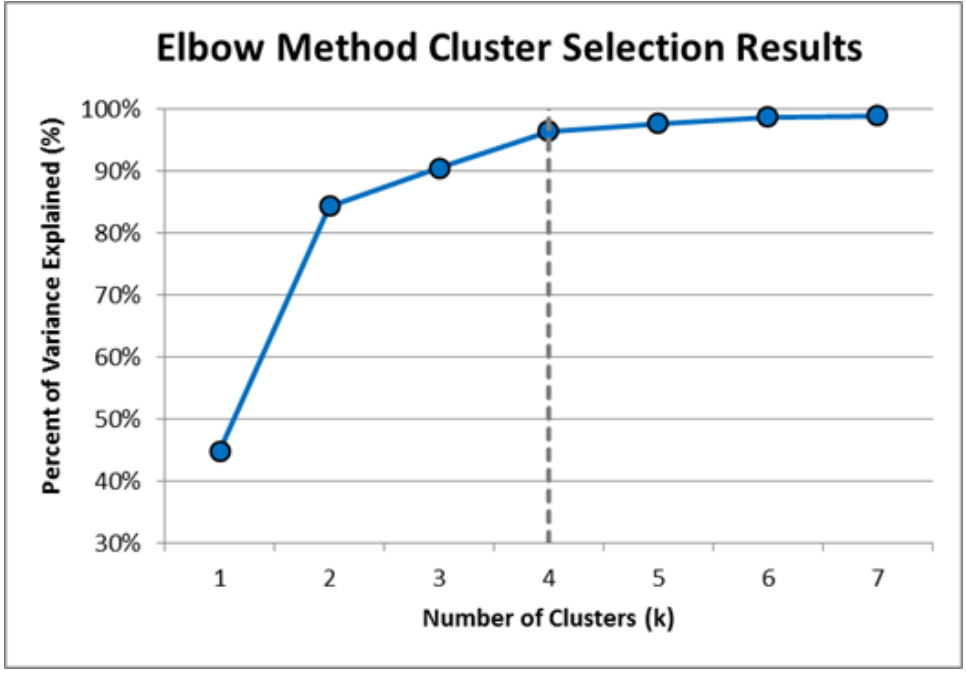




\section{Clustering Analysis}

\section{- Trip level k-medoid clustering analysis results}
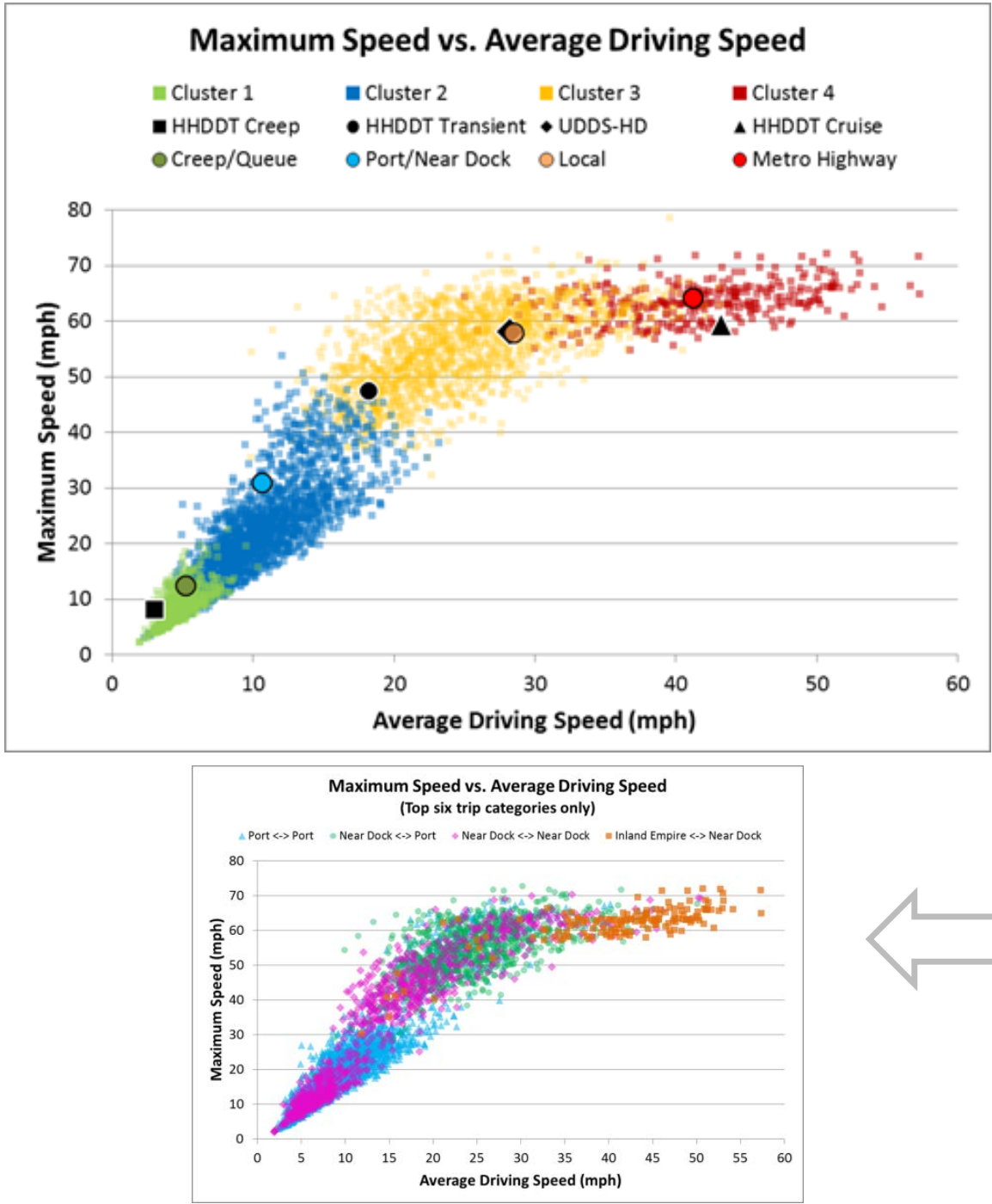

NATIONAL RENEWABLE ENERGY LABORATORY

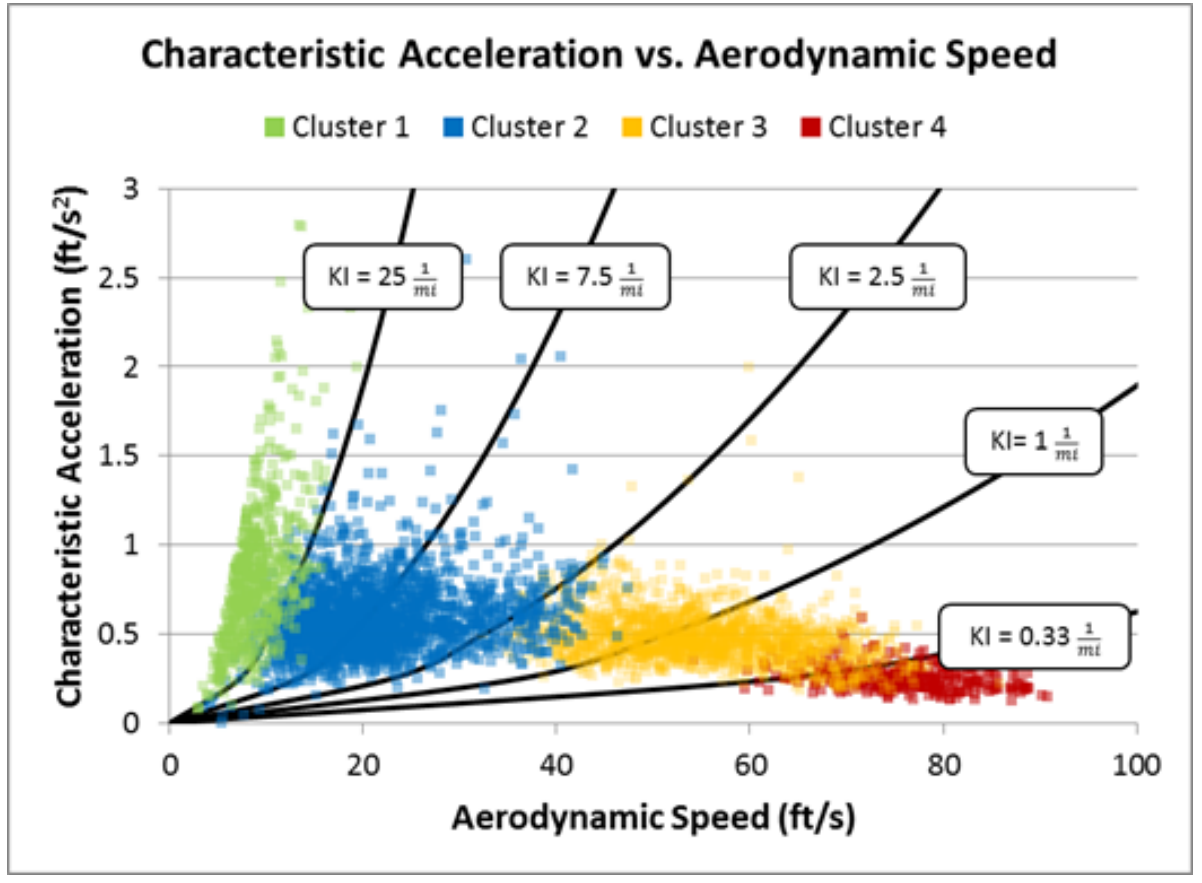

Previously shown geospatial trip classification for comparison 


\section{Clustering Results - Statistics}

- Trip-level statistics by cluster with standard deviation

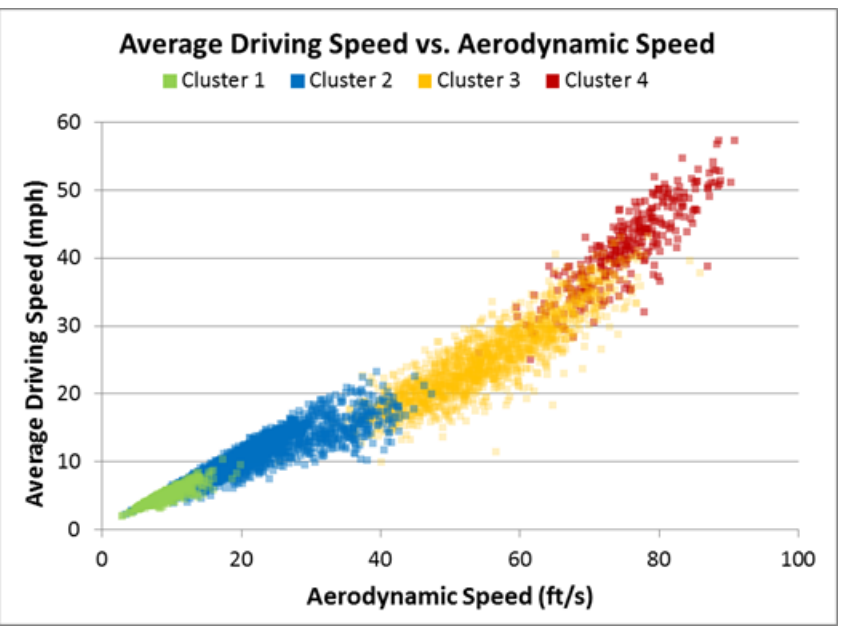

\begin{tabular}{|c|c|c|c|c|c|c|c|c|}
\hline \multirow{2}{*}{\begin{tabular}{|l|} 
Cluster \\
NREL Custom \\
Cycle \\
\end{tabular}} & Cluster 1 & $\sigma$ & Cluster 2 & $\sigma$ & Cluster 3 & $\sigma$ & Cluster 4 & $\sigma$ \\
\hline & \multicolumn{2}{|c|}{ Creep/Queue } & \multicolumn{2}{|c|}{ Port/Near Dock } & \multicolumn{2}{|c|}{ Local } & \multicolumn{2}{|c|}{ Metro Highway } \\
\hline $\begin{array}{l}\text { Number of Trips in } \\
\text { Cluster }\end{array}$ & 625 & N/A & 1874 & N/A & 1551 & N/A & 314 & N/A \\
\hline $\begin{array}{l}\text { Average Trip Length } \\
\text { (mi) }\end{array}$ & 0.12 & 0.13 & 1.06 & 0.92 & 11.05 & 6.43 & 54.93 & 25.39 \\
\hline $\begin{array}{l}\text { Average Driving } \\
\text { Speed (mph) }\end{array}$ & 4.90 & 1.20 & 10.76 & 3.38 & 24.81 & 5.74 & 41.76 & 6.08 \\
\hline $\begin{array}{l}\text { Average Total } \\
\text { Speed (mph) }\end{array}$ & 0.94 & 0.70 & 4.33 & 2.87 & 14.74 & 6.61 & 32.53 & 8.34 \\
\hline Average Total Stops & 2.83 & 3.17 & 4.54 & 4.15 & 13.73 & 9.53 & 14.97 & 11.38 \\
\hline $\begin{array}{l}\text { Average Stops per } \\
\text { Mile }\end{array}$ & 20.15 & 15.57 & 5.53 & 3.97 & 1.41 & 0.92 & 0.28 & 0.18 \\
\hline \begin{tabular}{|l|} 
Average Maximum \\
Speed (mph)
\end{tabular} & 8.81 & 3.03 & 22.72 & 8.64 & 54.89 & 7.21 & 63.44 & 3.40 \\
\hline $\begin{array}{l}\text { Average Kinetic } \\
\text { Intensity }(1 / \mathrm{mi}) \\
\end{array}$ & 55.10 & 22.17 & 8.84 & 6.45 & 0.94 & 0.50 & 0.24 & 0.09 \\
\hline $\begin{array}{l}\text { Average } \\
\text { Aerodynamic Speed } \\
(\mathrm{ft} / \mathrm{s})\end{array}$ & 9.10 & 2.52 & 21.82 & 7.60 & 54.91 & 9.24 & 75.63 & 6.53 \\
\hline \begin{tabular}{|l|} 
Average \\
Characteristic \\
Acceleration $\left(\mathrm{ft} / \mathrm{s}^{2}\right)$
\end{tabular} & 0.82 & 0.43 & 0.60 & 0.27 & 0.48 & 0.14 & 0.25 & 0.07 \\
\hline $\begin{array}{l}\text { Percent of Zero } \\
\text { Speed Time (\%) }\end{array}$ & $81 \%$ & $13 \%$ & $61 \%$ & $21 \%$ & $42 \%$ & $18 \%$ & $22 \%$ & $14 \%$ \\
\hline
\end{tabular}




\section{Clustering Results - Statistics}

- Distribution of distance, trips, operating time, and fuel consumption by cluster

- Clusters named generically based on composition of trip behavior and location.

\begin{tabular}{|l|c|c|c|c|}
\cline { 2 - 5 } \multicolumn{1}{c|}{} & Cluster 1 & Cluster 2 & Cluster 3 & Cluster 4 \\
\cline { 2 - 5 } \multicolumn{1}{c|}{} & $\begin{array}{c}\text { Creep/ } \\
\text { Queue }\end{array}$ & $\begin{array}{c}\text { Port/ } \\
\text { Near Dock }\end{array}$ & Local & $\begin{array}{c}\text { Metro } \\
\text { Highway }\end{array}$ \\
\hline Distance & $0.2 \%$ & $5.6 \%$ & $48.8 \%$ & $45.3 \%$ \\
\hline Number of Trips & $14.4 \%$ & $43.2 \%$ & $35.7 \%$ & $6.7 \%$ \\
\hline Operating Time & $4.2 \%$ & $23.0 \%$ & $53.3 \%$ & $19.5 \%$ \\
\hline Fuel & $1.0 \%$ & $10.4 \%$ & $49.5 \%$ & $39.2 \%$ \\
\hline
\end{tabular}




\section{Custom Drive Cycles by Cluster}

- Using NREL's DRIVE tool, statistically representative drive cycles were created for each cluster

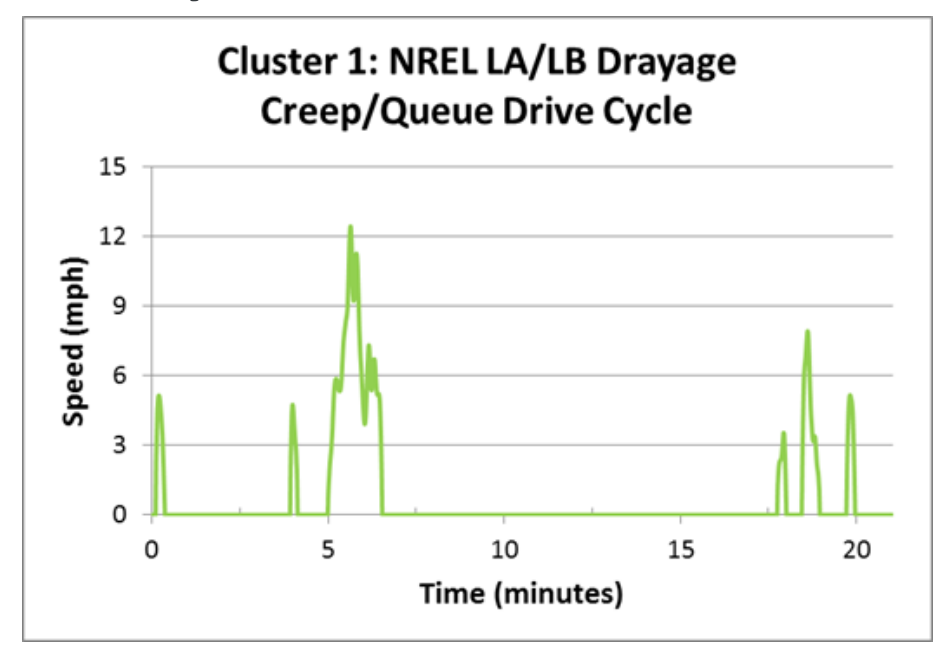

Cluster 2: NREL LA/LB Drayage Port/Near

Dock Drive Cycle
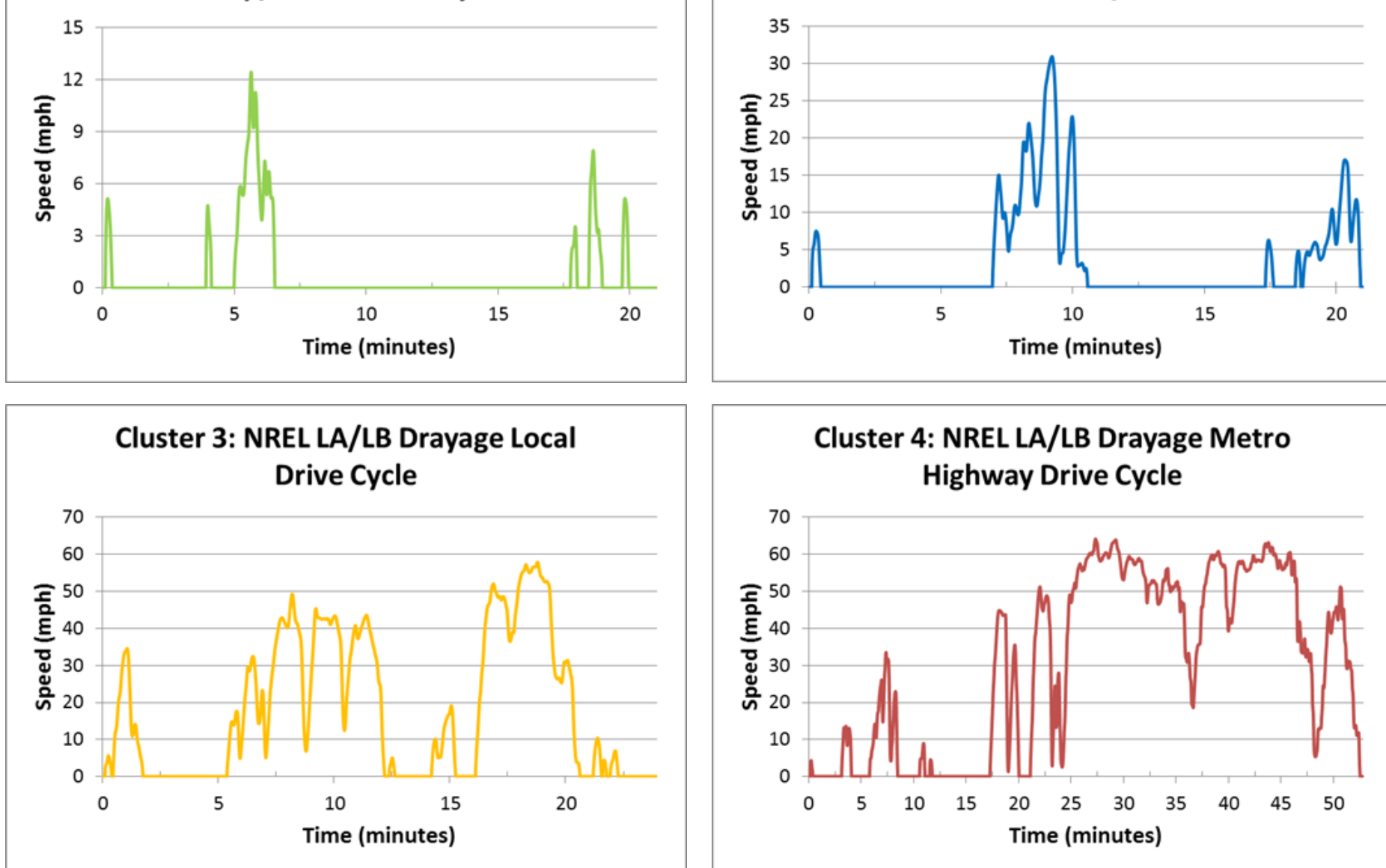

DRIVE: Drive-Cycle Rapid Investigation, Visualization, and Evaluation (http://www.nrel.gov/transportation/drive.html) 


\section{Custom Drive Cycles by Cluster}

- Drive cycle metrics from custom representative cycles

\begin{tabular}{|c|c|c|c|c|}
\hline Cluster & Cluster 1 & Cluster 2 & Cluster 3 & Cluster 4 \\
\hline NREL Custom Cycle & $\begin{array}{l}\text { Creep/ } \\
\text { Queue }\end{array}$ & $\begin{array}{c}\text { Port/ } \\
\text { Near Dock }\end{array}$ & Local & $\begin{array}{c}\text { Metro } \\
\text { Highway }\end{array}$ \\
\hline Drive Cycle Length (mi) & 0.26 & 1.17 & 7.12 & 26.66 \\
\hline Drive Cycle Duration (minutes) & 22.17 & 21.02 & 23.93 & 52.82 \\
\hline Average Driving Speed (mph) & 5.20 & 10.61 & 28.53 & 41.23 \\
\hline Average Total Speed (mph) & 0.70 & 3.34 & 17.86 & 30.29 \\
\hline Total Stops & 6 & 5 & 9 & 7 \\
\hline Stops per Mile & 23.33 & 4.27 & 1.26 & 0.26 \\
\hline Maximum Speed (mph) & 12.46 & 30.98 & 57.90 & 64.17 \\
\hline Average Kinetic Intensity (1/mi) & 15.89 & 3.79 & 0.69 & 0.24 \\
\hline Average Aerodynamic Speed (ft/s) & 10.40 & 25.88 & 59.30 & 75.07 \\
\hline Average Characteristic Acceleration $\left(\mathrm{ft} / \mathrm{s}^{2}\right)$ & 0.33 & 0.48 & 0.46 & 0.25 \\
\hline
\end{tabular}

Cluster 1: NREL LA/LB Drayage

Creep/Queue Drive Cycle

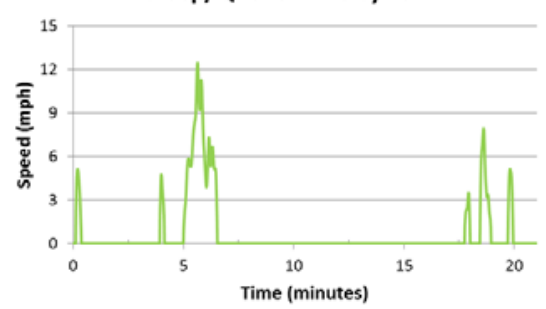

Cluster 2: NREL LA/LB Drayage Port/Near Dock Drive Cycle

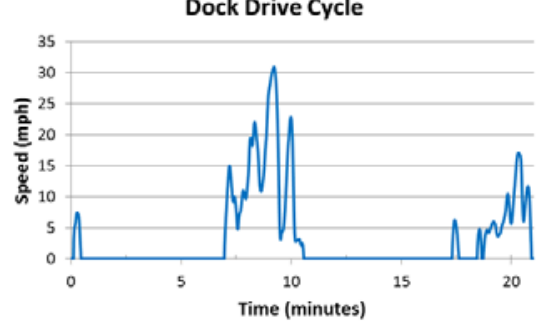

Cluster 3: NREL LA/LB Drayage Local Drive Cycle

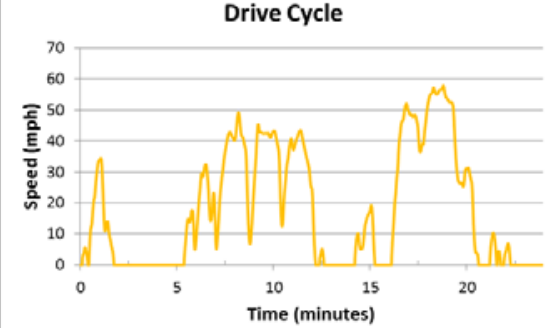

Cluster 4: NREL LA/LB Drayage Metro Highway Drive Cycle

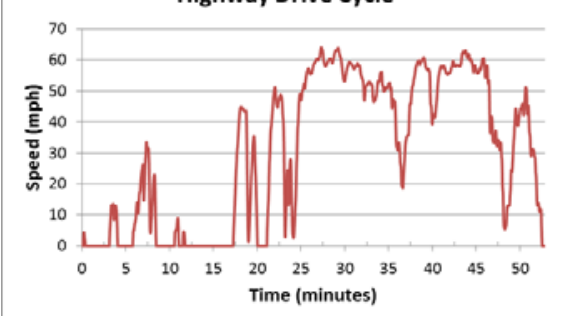




\section{NREL POLA/POLB Composite Drive Cycle}

- Cycles can be run independently or combined into a single composite cycle

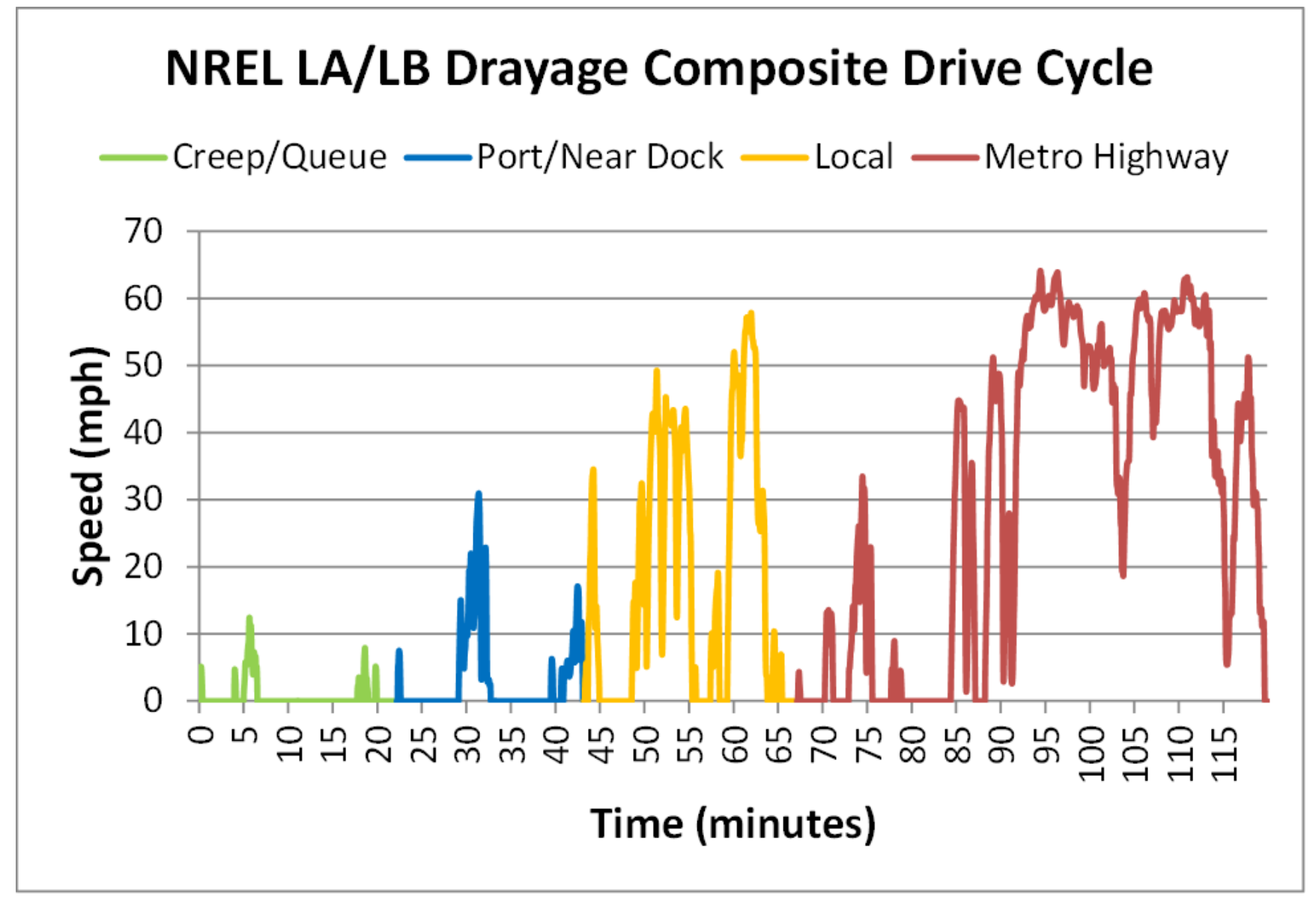


- Apply same methodology to other vocations to identify \& characterize unique operating modes

o Package delivery trucks

o School buses

o Transit buses

o Utility aerial trucks

o Refuse trucks
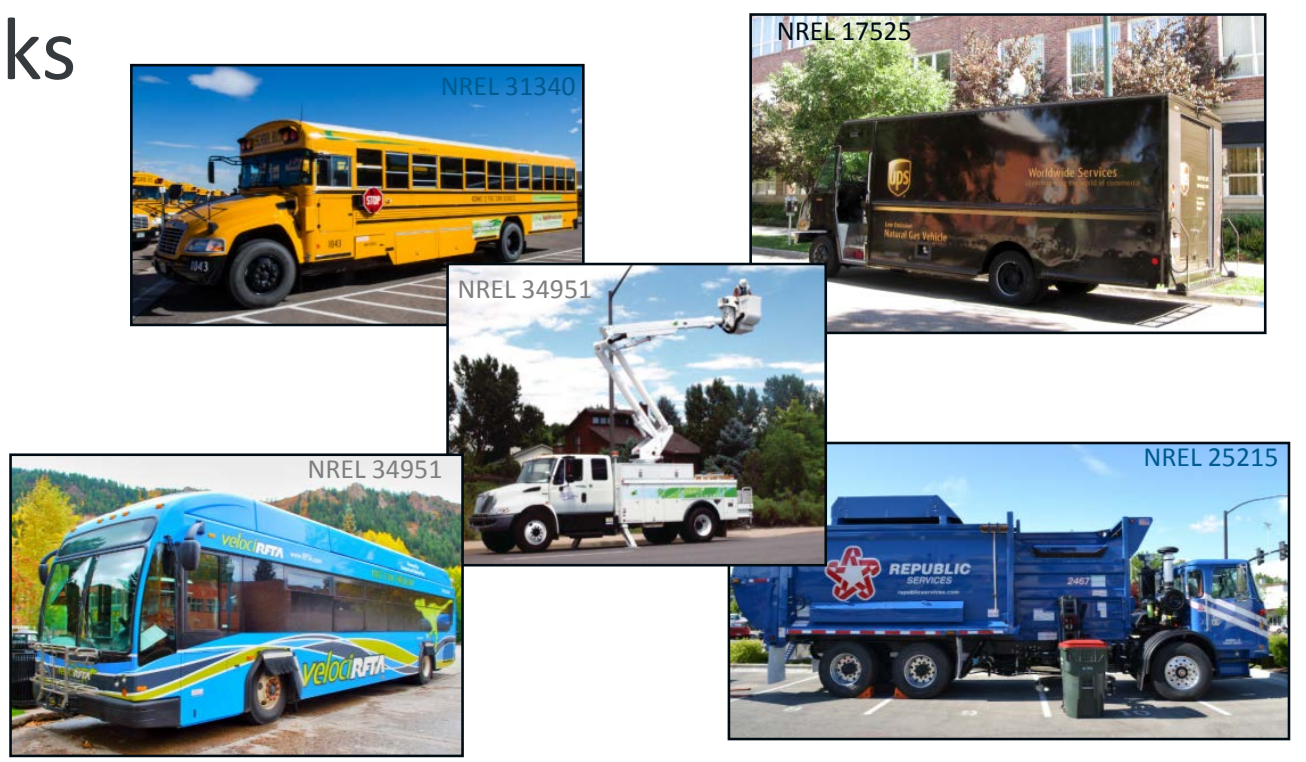

- Create representative drive cycles for each unique mode identified and make publically available 


\section{Applying Drive Cycle Data - NREL DriveCAT}

\section{Objectives}

- Provide a common, publicly available, easy-to-use site for standard and custom drive cycles for MD / HD vehicles

- Capture, quantify and compare drive cycle variation across the spectrum of MD / HD vocations

- Allow users to download raw time series data of drive cycles for their own use

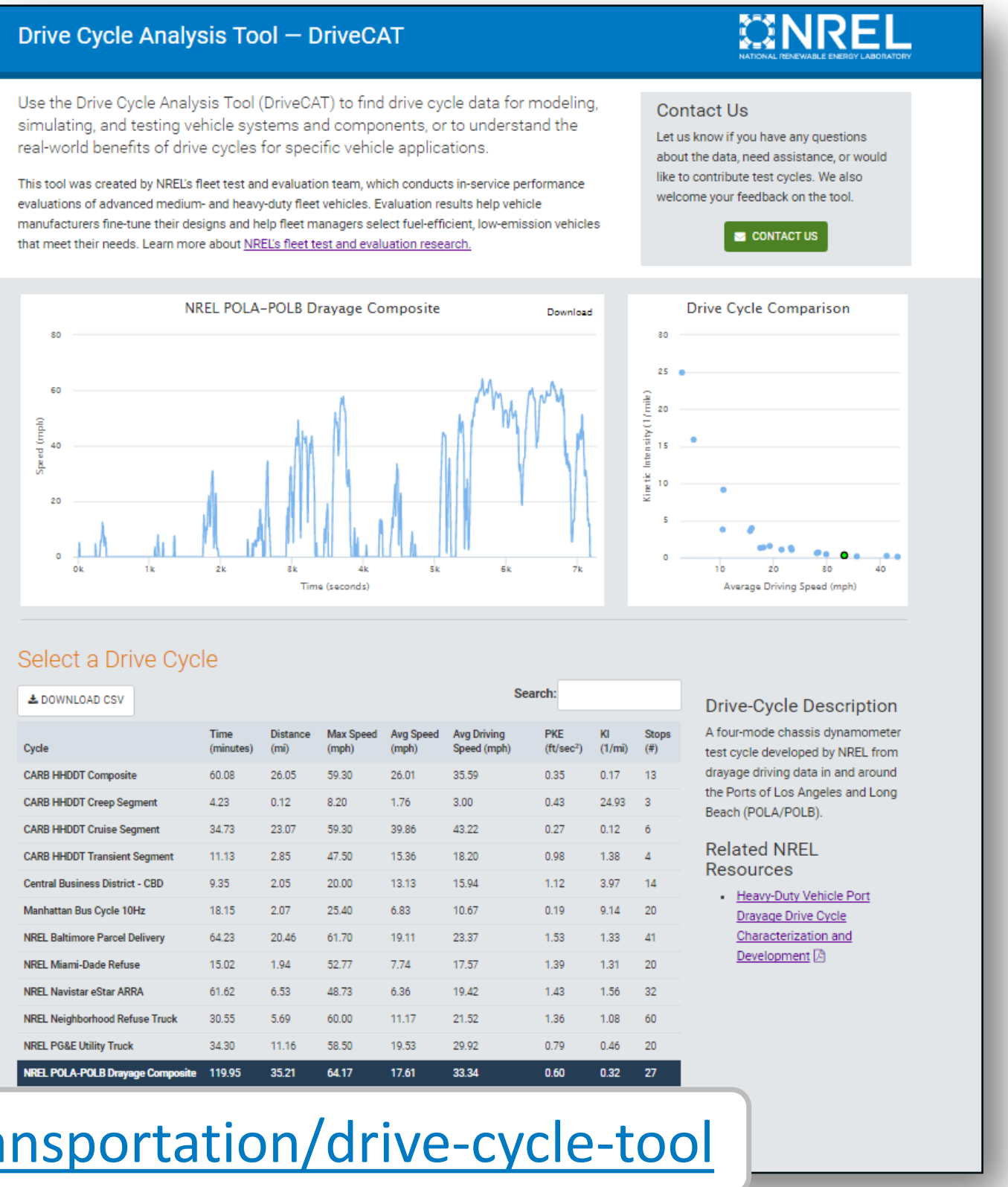

www.nrel.gov/transportation/drive-cycle-tool 


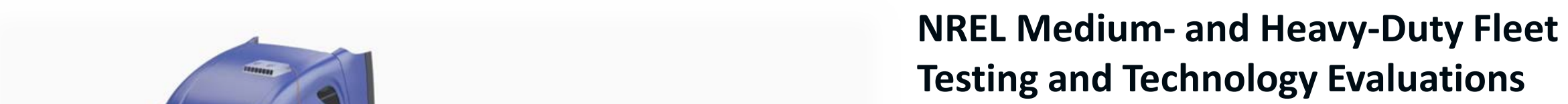
Testing and Technology Evaluations

Supported by:

U.S. Department of Energy Vehicle Technologies Office Vehicle Systems Program

- Lee Slezak and David Anderson

Questions / Comments / Collaboration:

Robert Prohaska Robert.Prohaska@NREL.gov

Kenneth Kelly (Task Leader) Kenneth.Kelly@NREL.gov

www.nrel.gov

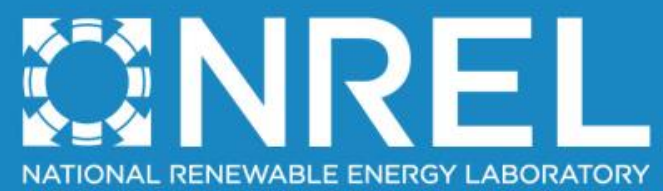

